\title{
15 \\ The Way of Doing Things
}

A newly hired factory worker was instructed by some workmates about her different tasks:

OK, this is the way you are supposed to do it, but this is the way that I actually do it because it is quicker (or safer, or less tiring). But don't let the supervisor see you doing it like that, or tell him that you worked it out for yourself. Don't say that I showed you.

That was not, of course, the way to do the job that had been decided by management.

(White, 1988) 